\title{
CT Diagnosis of COVID-19 Based On the Imaging Signs of Pulmonary Lobular
}

\author{
deli zhao ${ }^{1}$, guokun wang ${ }^{1}$, peng chen $^{1}$, Shanshan $\mathrm{Yu}^{1}$, Haibo Wang ${ }^{1}$, Xin Wang $^{1}$, and \\ jinling zhang ${ }^{1}$
}

${ }^{1}$ Affiliation not available

May 6, 2020

\begin{abstract}
Objectives To study the CT diagnosis of COVID-19 that was based on the imaging features of pulmonary lobular structure. Materials and methods After institutional review board approval and informed consent, 139 cases with suspected COVID-19 who undergone $\mathrm{CT}$ were retrospectively analyzed. Based on the anatomical structure of pulmonary lobule, the imaging features of pulmonary lobular structure on CT images was defined and built simulation diagrams serve as a unified standard. The imaging performance of confirmed 103 cases was evaluated for diagnosis of COVID-19. Results Of these139 cases, 103 cases were confirmed for COVID-19, 36 cases were confirmed for other diseases. Of the 103 COVID-19 cases, 82(79.61\%) cases were typical cases and $21(20.39 \%)$ cases were atypical cases. $65(63.11 \%$ ) cases were in the early or advanced stage, $26(25.24 \%)$ cases were in the severe or recovery stage, and 12(11.65\%)were in multiple stages. The scores of pulmonary lobular structure in typical COVID-19 (3.42 \pm 1.25$)$ were significant different compared with atypical COVID-19 (1.51 $\pm 1.34, \mathrm{P}<0.001)$. The inter-observer agreement for the score of pulmonary lobular structure was excellent (kappa $=0.863, \mathrm{P}<0.05$ ). Conclusion To recognize the CT common imaging signs of pulmonary lobular structure in COVID-19 can deepen the understanding of COVID-19 from the basis of image anatomy, and screen suspected cases and provide imaging basis for diagnosis and differential diagnosis.
\end{abstract}

\section{INTRODUCTION}

According to the latest guidelines for diagnosis and treatment of Novel coronavirus pneumonia(COVID-19) by the General Office of the National Health Commission of China, the use of fluorescent reverse transcriptase polymerase chain reaction(RT-PCR) to detect nucleic acids of the coronavirus is the gold standard for molecular diagnosis of COVID-19(1,2), but nucleic acid detection has several limitations, including the need for high specificity of primer sets, low sensitivity, and the length of testing period. In comparison, $\mathrm{CT}$ (Computed tomography) imaging offers a simple, fast, and highly sensitive means to elucidate the extent and nature of lung tissue in COVID-19. After analyzing the CT images of confirmed COVID-19, we noticed that the signs of pulmonary lobular structures were commonly associated with all stages of COVID-19. Recognizing the underlying pulmonary lobular structural image signs can enable us to understand the typical or atypical CT characteristics of COVID-19, so as to provide reliable imaging diagnosis and differential diagnosis for clinical early diagnosis and prognosis judgment(3).

\section{MATERIALS AND METHODS}

\section{Population and CT image data acquisition}

We retrospectively reviewed 139 cases that were collected from January 26 to February 10, 2020. The data characteristics of all patients are shown in Table 1.Patients were examined using the conventional CT(16slice or 64-slice CT) machines. Scanning parameters and tube voltage were set to automatic mode for chest. Images were reconstructed using the best electronic voltage measured, with 5 millimeter slice thickness, and 
5 millimeter slice interval. This study is approved by our Institutional Review Board, which is waived the requirement for patients' informed consent referring to the CIOMS guideline.

\section{CT images analysis}

\section{Classification of pulmonary lobular structural imaging signs}

To define the imaging features of pulmonary lobular structure on CT images, then building simulation schematics to strengthen cognition and serve as a unified standard for evaluation (Fig.1, 2). The pulmonary lobular structural imaging signs are defined as a set of CT imaging of pulmonary lobules and its related structures, which are typified by five major categories: I Lobular size sign, II Fusion lobular sign, III interlobular septal thickening sign, IV intralobular interstitial thickening sign, V intralobular vascular thickening sign (Fig. 2).

\section{Definition of typical CT features of COVID-19}

Combined with the typical imaging diagnostic standards of COVID-19 that were reported by domestic imaging diagnosis guidelines and some literatures (16-18), We define the cases with the following three CT features as typical COVID-19 cases and the others as atypical cases in this study. These three features are as follows: First, density: Multiple GGOs or consolidations are present. Secondly, size: Pulmonary lobular or fusion lobule. Thirdly, distribution: Subpleural distribution with major axis nearly parallel to pleura and perpendicular to the bronchovascular bundle.

\section{Score standard for evaluation pulmonary lobular structure}

Pulmonary lobular structure scores for all confirmed cases which include lobular size sign (1 score), fusion lobular sign (1 score), interlobular septal thickening sign (1 score), intralobular interstitial thickening sign (1 score) and intralobular vascular thickening sign (1 score). Two senior radiologists with more than 10 years of experience each independently based on the imaging signs of pulmonary lobular structure and assessed all the cases which include confirmed cases of COVID-19 (typical,atypical and multi-stage ) and confirmed cases of non-COVID-19.

\section{Statistic analysis}

Statistical analyses were performed with the SPSS software (version 22.0, IBM).Count index was presented as percentage, and all data were presented as means and standard deviation (mean $\pm \mathrm{SD}$ ). Independent sample t-test or Chi square test was used. Inter-observer agreement for the subjective image quality was estimate using kappa statistic. $\mathrm{P}<0.05$ was considered statistically significant.

\section{RESULTS}

Of these 139 cases, 103 cases were confirmed for COVID-19 and 36cases were confirmed other diseases(including other pneumonia, pulmonary hemorrhage, pulmonary edema and traumatic wet lung). Of the 103 COVID-19 cases, 82(79.61\%) cases were typical cases and 21(20.39\%)cases were atypical cases. $65(63.11 \%)$ cases were in the early or advanced stage, 26(25.24\%) cases were in the severe or recovery stage, and $12(11.65 \%)$ were in multiple stages. Two radiologists scored the imaging signs of pulmonary lobular structure of COVID-19 and other diseases. By comparing the image features of typical cases and atypical cases, there was statistical difference between the size $<3 \mathrm{~cm}$, the density of GGO and consolidation, but there was no statistical difference between the size $>3 \mathrm{~cm}$ and the density of mixed GGO, there is significant statistical difference between typical cases and atypical cases of distribution and location. The scores of pulmonary lobular structure in typical COVID-19 (3.42+-1.25) were significant different compared with atypical COVID-19 (1.51+-1.34, $\mathrm{P}<0.001)$. The inter-observer agreement for the score of pulmonary lobular structure was excellent $(\mathrm{kappa}=0.863, \mathrm{P}<0.05)$. The diagnosis of typical CT features (Fig. 3) and atypical CT features (Fig. 4) in COVID-19 are shown in table 2. The evaluation results of dynamic multi-stage images (Fig. 5) and differential diagnosis with non- COVID-19(Fig. 6) based on the image signs of pulmonary lobular structure are shown in and table 3. 


\section{DISCUSSION}

Pulmonary lobule is the basic unit of pulmonary anatomic function, which has been playing an important role in HRCT. The imaging features of pulmonary lobular structure run through all stages of the image performance of COVID-19. According to the anatomical differences of pulmonary lobules, Fleischner and Heitzman proposed that the lung can be divided into peripheral pulmonary cortex and central pulmonary medulla $(4,5)$. Webb put forward the concept of cortical lobule, highlighting the anatomical characteristics of pulmonary cortical lobule. The characteristics of the pulmonary cortical lobule also lay the anatomical basis for the distribution of COVID-19 under the pleura $(6,7)$. Therefore, it is critical to discern the characteristics of lobules and their distribution for accurate diagnosis of COVID-19.

The CT features of viral pneumonia, such as lobular size, fusion lobule, thickening of intralobular interstitium and interlobular septum, that have been respectively reported in the related literatures of influenza and SARS(8-12). In particular, the novel coronavirus pneumonia has a virus homology with SARS. The image of COVID-19 is highly similar to the lobular image feature reported by SARS(13-16). Therefore, the evaluation method and scoring standard of five categories of pulmonary lobular structure image based on the anatomical structure of pulmonary lobule were proposed for the first time in this study. The lobular size sign in COVID19 often shows GGO or consolidation of 1-3centimeters, which is related to the size study of SARS by Wong (13). The fusion lobular sign is defined as the shape of wedge-shaped, fan-shaped, irregular or bat wing with the size between 3 centimeters and lung lobe, which does not follow the lung segments or lobe. In the early stage, the interlobular septal thickening sign is generally not obvious, only to make the edge of the lesion clearer, and transit to grid shape in the advanced or the recovery stage. The interlobular interstitial thickening sign likes "paving stone sign" which is very characteristic, and is very specific in the diagnosis of COVID-19. The interlobular vascular thickening sign is more common in the peripheral pulmonary cortex lobular blood vessels, particularly in the early stage of COVID-19.

From the perspective of image thinking, CT images of different types COVID-19 mainly include typical and atypical CT features, and the evaluation of dynamic stages. In this study, combined with the typical imaging diagnostic standards of COVID-19 that were reported by domestic imaging diagnosis guidelines and some literatures (16-18), as well as a summary of 103 confirmed cases, 82(79.61\%) single-stage and $12(11.65 \%)$ multi-stage cases of the typical COVID-19 have three characteristics. These three typical performances of COVID-19 are consistent with some reports (19-21), but the ideas and concerns of authors are based on the image signs of pulmonary lobular structure, and the analysis from density, size and distribution is more in line with the traditional image thinking mode. As a new infectious disease, COVID-19 has not been fully recognized so far. In our study, we found that the image manifestations of atypical cases are mostly GGO or consolidation, including single or mixed GGO, thickening of bronchovascular bundle with peripheral GGO, mixed GGO in lobes or segments, and halo sign or anti halo sign in a few cases. These findings are consistent with the relevant literature (20-22).

In this study, there are two types of differential diagnosis of COVID-19: infectious disease(other viral pneumonia, bacterial pneumonia, mycoplasma infection, cryptogenic organizing pneumonia, eosinophilic pneumonia) and non-infectious disease(pulmonary hemorrhage, pulmonary edema and traumatic wet lung). To some extent, the imaging manifestations of these diseases may overlap with COVID-19, so it is great significance to combine the imaging signs of pulmonary lobular structure for differential diagnosis between COVID-19 and non-COVID-19. However, there are some limitations in this study, such as the small number of non-COVID19 cases and the uncertain differential diagnosis. This is also the research direction of the next step after increasing the number of samples.

\section{CONCLUSION}

In this study we found that recognize the common imaging signs of pulmonary lobular structure in COVID-19 can deepen the understanding of COVID-19 from the basis of image anatomy, and provide reliable imaging diagnosis and differential diagnosis basis for early clinical diagnosis.

\section{References}


1. Huang C, Wang Y, Li X, et al. Clinical features of patients infected with 2019 novel coronavirus in Wuhan, China[J]. Lancet.2020;S0140-6736(20)30183-30185.

2.Sohrabi C, Alsafi Z, O'Neill N, et al.World Health Organization declares Global Emergency:Areviewofthe2019NovelCoronavirus(COVID-19)[J].International Journal of Surgery.2020;https://doi.org/10.1016/j.ijsu.2020.02.034.

3. Lee, KS. Pneumonia Associated with 2019 Novel Coronavirus: Can Computed Tomographic Findings Help Predict the Prognosis of the Disease?[J].Korean J Radiol. 2020;21(3):257-258.

4.Fleischner, FG. The butterfly pattern of acute pulmonary edema[J].Am J Cardiol. 1967;20(1):39-46.

5.Heitzman ER, Markarian B, Berger I, et al.The secondary pulmonary lobule: a practical concept for interpretation ofchest radiographs. I. Roentgen anatomy of the normal secondary pulmonarylobule[J].Radiology.1969;93(3):507-512.

6.Webb WR. High-resolution CT of the lung parenchyma[J]. Radiol Clin North Am. 1989;27(6):1085-1097.

7. Webb WR, Stein MG, Finkbeiner WE, et al. Normal and diseased isolated lungs: high-resolution CT[J]. Radiology.1988;166(1 Pt 1):81-87.

8. Chan MS, Chan IY, Fung KH, et al.High-resolution CT findings in patients with severe acute respiratorysyndrome: a pattern-based approach[J]. AJR Am J Roentgenol. 2004,182(1):49-56.

9. Antonio GE, Wong KT, Hui DS, et al.Thin-section CT in patients with severe acute respiratory syndrome followinghospital discharge: preliminary experience[J]. Radiology.2003;228(3):810-815.

10.Franquet T. Imaging of pulmonary viralpneumonia[J].Radiology.2011;260(1):18-39.

11. Koo HJ, Lim S, Choe J, et al.Radiographic and CT Features of ViralPneumonia[J]. Radiographics.2018;38(3):719-739.

12. Rao TN, Paul N, Chung T, et al.Valueof CT in assessing probable severe acute respiratory syndrome[J]. AJR Am JRoentgenol.2003;181(2):317-319.

13.Wong KT, Antonio GE, Hui DS, et al.Thin-section CT of severe acute respiratory syndrome: evaluation of 73 patients exposed to or with the disease[J]. Radiology. 2003;228(2):395-400.

14.Ketai L; Paul NS; Wong KT. Radiologyof severe acute respiratory syndrome (SARS): the emerging pathologic-radiologiccorrelates of an emerging disease[J]. J Thorac Imaging.2006;21(4):276-283.

15. Muller NL, Ooi GC, Khong PL, et al. High-resolution CT findings of severe acute respiratory syndrome atpresentation and after admission[J].AJR.2004;182:239-244.

16. Pan Y, Guan H, Zhou S, et al. Initial CT findings and temporal changes in patients with the novel coronavirus pneumonia (2019-nCoV): a study of 63 patients in Wuhan, China[J]. Eur Radiol.2020;Feb13 DOI: $10.1007 / \mathrm{s} 00330-020-06731-\mathrm{x}$.

17. Pan F, Ye T, Sun P, et al. Time Course of Lung Changes On Chest CT During Recovery From 2019 Novel Coronavirus (COVID-19) Pneumonia[J]. Radiology. 2020;Feb 13:200370.

18. Xiong Y,Sun D,Liu Y, et al.Clinical and High-Resolution CT Features of the COVID19 Infection: Comparison of the Initial and Follow-up Changes[J].Invest Radiol.2020;Mar 3.PMID: 32134800.DOI: 10.1097/RLI.0000000000000674.

19. Habibzadeh P, Stoneman EK.The Novel Coronavirus: A Bird's Eye View[J]. Int J Occup Environ.2020;Med 11(2):65-71.

20. Zu ZY, Jiang MD, Xu PP, et al. Coronavirus Disease 2019 (COVID-19): A Perspective from China[J]. Radiology.2020;Feb 21:200490. 
21. Chung M, Bernheim A, Mei X, et al. CT Imaging Features of 2019 Novel Coronavirus (2019-nCoV)[J]. Radiology.2020;Feb 4:200230.

22. Ye Z, Zhang Y,Wang Y,et al.Chest CT manifestations of new coronavirus disease 2019 (COVID-19): a pictorial review[J]. Eur Radiol.2020;Mar 19 https://doi.org/10.1007/s00330-020-06801-0.

Tables

Table 1. Comparison baseline characteristics between COVID-19 cases with non-OVID-19 cases

\begin{tabular}{llll}
\hline Characteristic & COVID-19 $(\mathrm{n}=103)$ & Non-OVID-19 $(\mathrm{n}=36)$ & P Value \\
Mean age(y) & $47.3 \pm 12.5$ & $42.5 \pm 13.2$ & 0.792 \\
$\begin{array}{l}\text { Sex } \\
\text { Male(n) }\end{array}$ & $63(61.17 \%)$ & $25(69.44 \%)$ & 0.375 \\
Female(n) & $40(38.83 \%)$ & $11(30.56 \%)$ & \\
Symptom and signs & & & \\
Pharyngeal discomfort & $10(9.71 \%)$ & $2(5.56 \%)$ & 0.675 \\
Fever & $85(82.52 \%)$ & $9(25.00 \%)$ & $<0.0001$ \\
Cough & $66(64.08 \%)$ & $17(47.22 \%)$ & 0.076 \\
Abdominal pain & $12(11.65 \%)$ & 0 & 0.036 \\
Temperature $(>37.3)$ & $98(95.15 \%)$ & $26(72.22 \%)$ & 0.001 \\
White blood cell count $(\times 109 / \mathrm{L})$ & & & \\
Normal/decreased & $85(82.52 \%)$ & $22(61.11 \%)$ & 0.009 \\
Increased & $18(17.48 \%)$ & $14(38.89 \%)$ & \\
C-rective protein $(\mathrm{mg} / \mathrm{L})$ increased & $87(84.47 \%)$ & $19(52.78 \%)$ & 0.0001 \\
\hline
\end{tabular}

Note: Date in parentheses is number of cases. Data are $\mathrm{n}(\%)$, mean (SD), $\mathrm{p}<0.05$. Increased means over the upper limit of the normal range and decreased means below the lower limit of the normal range.

Table 2. Comparison CT image characteristics between typical cases with atypical cases in COVID-19

\begin{tabular}{llll}
\hline Characteristic & Typical $(\mathrm{n}=82)$ & Atypical $(\mathrm{n}=21)$ & P Value \\
Size & & & \\
Small nodule $(<1 \mathrm{~cm})$ & $2(2.44 \%)$ & $5(23.81 \%)$ & 0.003 \\
Lobule (1 to 3cm) & $38(46.34 \%)$ & $3(14.29 \%)$ & 0.007 \\
3cm to lobe & $42(51.22 \%)$ & $13(61.90 \%)$ & 0.381 \\
Distribution and location & & & \\
Focal & 0 & $9(42.86 \%)$ & $<0.01$ \\
Multi-focal/diffuse & 82 & $12(57.14 \%)$ & $<0.01$ \\
Airway & 0 & $7(58.33 \%)$ & $<0.01$ \\
Subpleural & 82 & 0 & $<0.01$ \\
Segmental/lobe & 0 & $5(41.67 \%)$ & $<0.01$ \\
Density & & & \\
Pure-GGO & $43(52.44 \%)$ & $4(19.05 \%)$ & 0.006 \\
Mix-GGO & $28(34.15 \%)$ & $9(42.86 \%)$ & 0.458 \\
Consolidation & $11(13.41 \%)$ & $8(38.10 \%)$ & 0.009 \\
Lobular structure scores & $3.42 \pm 1.25$ & $1.51 \pm 1.34$ & $<0.0001$ \\
\hline
\end{tabular}

Note: Number in parentheses are percentages, Data are n (\%), mean (SD), $\mathrm{p}<0.05$. 
Table 3. Evaluation of imaging features of pulmonary lobular structure between COVID-19 and non- COVID-19

\begin{tabular}{llllll}
\hline Characteristic & Lobular size sign & $\begin{array}{l}\text { Fusion lobular } \\
\text { sign }\end{array}$ & $\begin{array}{l}\text { Interlobular } \\
\text { septal thickening }\end{array}$ & $\begin{array}{l}\text { Intralobular } \\
\text { vascular } \\
\text { thickening }\end{array}$ & $\begin{array}{l}\text { Intralobular } \\
\text { interstitial } \\
\text { thickening }\end{array}$ \\
COVID-19 & & & & ++ & ++ \\
Single stage & +++ & ++ & + & + & + \\
Multi-stage & + & ++ & + & + & + \\
Early stage & ++ & ++ & + & ++ & ++ \\
Advanced & ++ & ++ & + & ++ & ++ \\
Stage & & +++ & ++ & + & ++ \\
Severe stage & + & ++ & + & + & + \\
Recovery stage & + & + & & & + \\
Non- & + & & & &
\end{tabular}

Note: Plus signs indicate the relative frequency of the findings from lowest $(+)$ to higest $(+++)$;shot signs indicate no findings in this study.

Figure Legend

Note In order to facilitate the reader's recognition of pulmonary lobular signs, different arrows in figure2-6 in this manuscript represent the different signs (green arrow shows lobular size sign, green double-headed arrow shows fusion lobular sign, black arrow shows lobular septal thickening, white arrow shows lobular interstitial thickening sign, red arrow shows lobular vascular thickening sign).

Figure 1 Schematic of lobular structure of axial CT. The reticular structure of the left mimics the distribution of pulmonary lobules, and the larger one is the cortex lobule and the smaller one is the medullary lobule. The right is an enlarged simulated lobular structure. Letter a refers to the interlobular septum, b refers to intralobular vessel, and c refers to the intralobular interstitium.

Figure 2 Schematics of the lobular structural characteristics that include five categories: Lobular size (a), fusion lobules (b), interlobular septal thickening (c), intralobular interstitial thickening (d), intralobular vascular thickening (e). a1-elimages are the actual CT axial images corresponding to the schematics.

Figure 3 The combined picture (a-f) is composed of typical cases of COVID-19. All the cases are consistent with the three characteristics which include multiple GGOs or consolidations, pulmonary lobular or fusion lobule size and subpleural distribution.

Figure 4 Six COVID-19 cases are selected to represent the five atypical CT features. a-b represent single GGO or consolidation, c-d represent halo sign or reverse-halo sign, e-f represent bronchovascular bundle thickening with peripheral GGO or consolidation.

Figures 5 a-c Early, advanced and recovery stages of one COVID-19 patient are shown. a. The size of the lobule in the early stage. b. Fusion lobules in the advanced stage. The intralobular interstitial and interlobular vascular thickened. c. Lobular septal thickening in the recovery stage. d-f. Advanced, severe and recovery stages of another COVID-19 patient are depicted. d. During the advanced stage, the area of right pulmonary fusion lobule is increased, and the new leisons with lobular size are seen in the left lung. e. Diffuse mosaic sign ("white lung") in severe stage. f. The interlobular septal thickening in the recovery stage.

Figure 6 a-f are differential diseases with COVID-19 in CT imaging respectively. Lobar pneumonia(a) is characterized by consolidation of lobar segments without obvious pulmonary lobular structure signs. Mycoplasma pneumonia (b) and Influenza pneumonia(c), both of them can be seen the sign of fusion lobule, but 
the mycoplasma pneumonia is distributed along the bronchovascular bundle. Pulmonary edema (d), there is obvious sign of interlobular septal thickening. Both traumatic wet lung (e) and pulmonary hemorrhage (f) show subpleural GGO, but there is no significant pulmonary lobular structure signs.

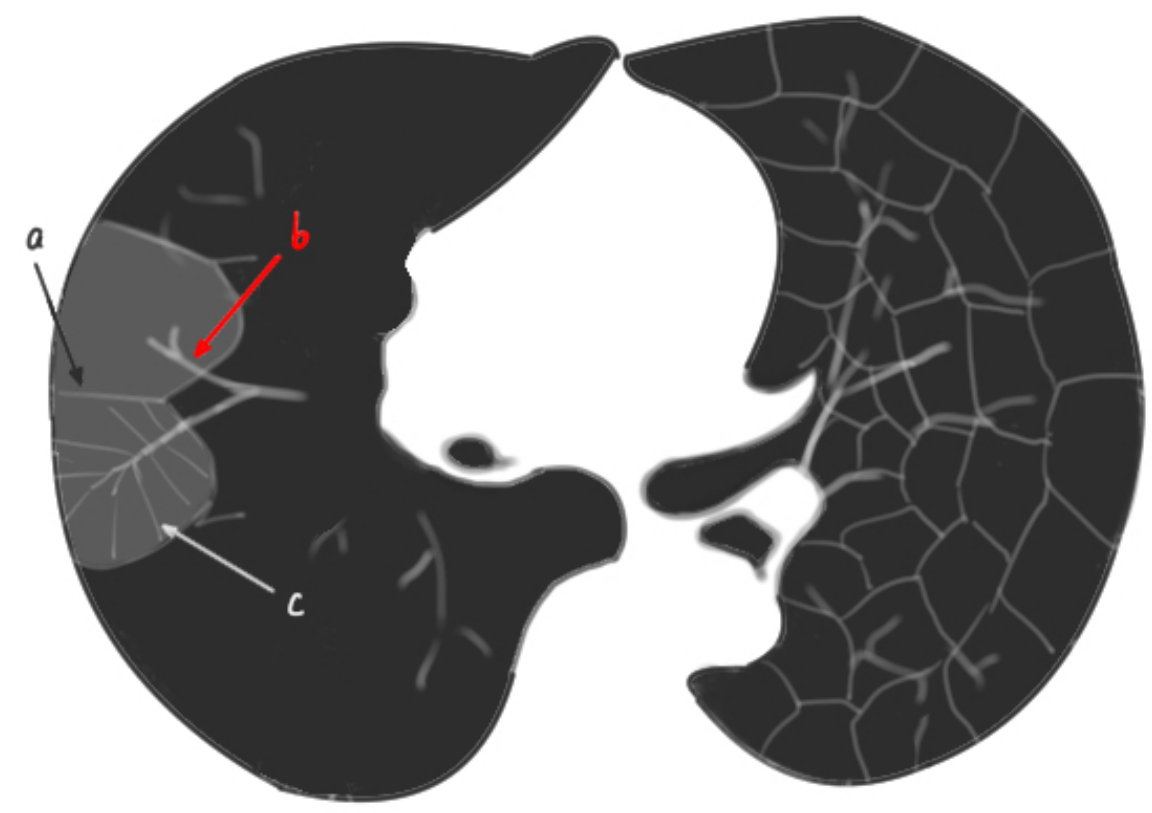




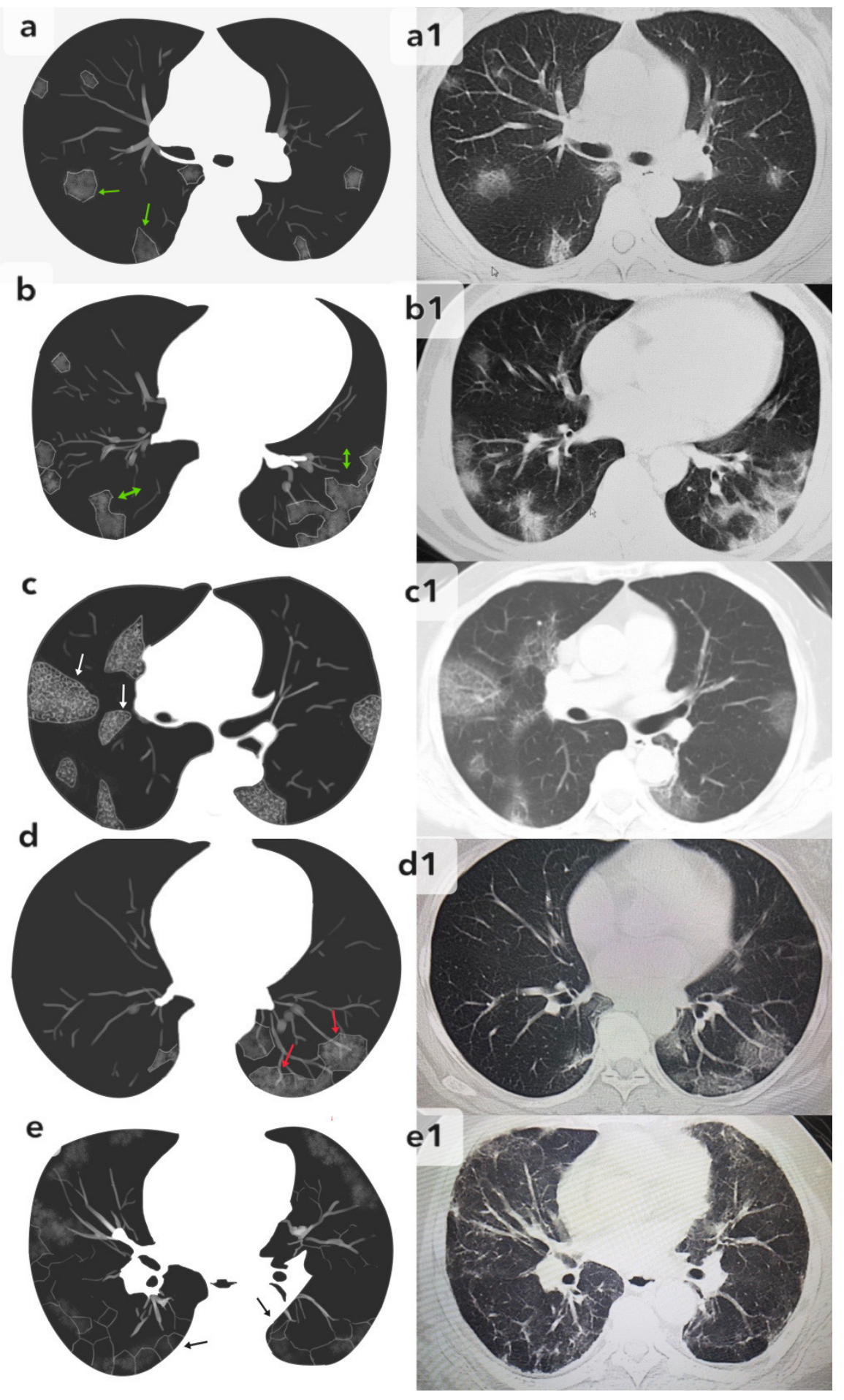



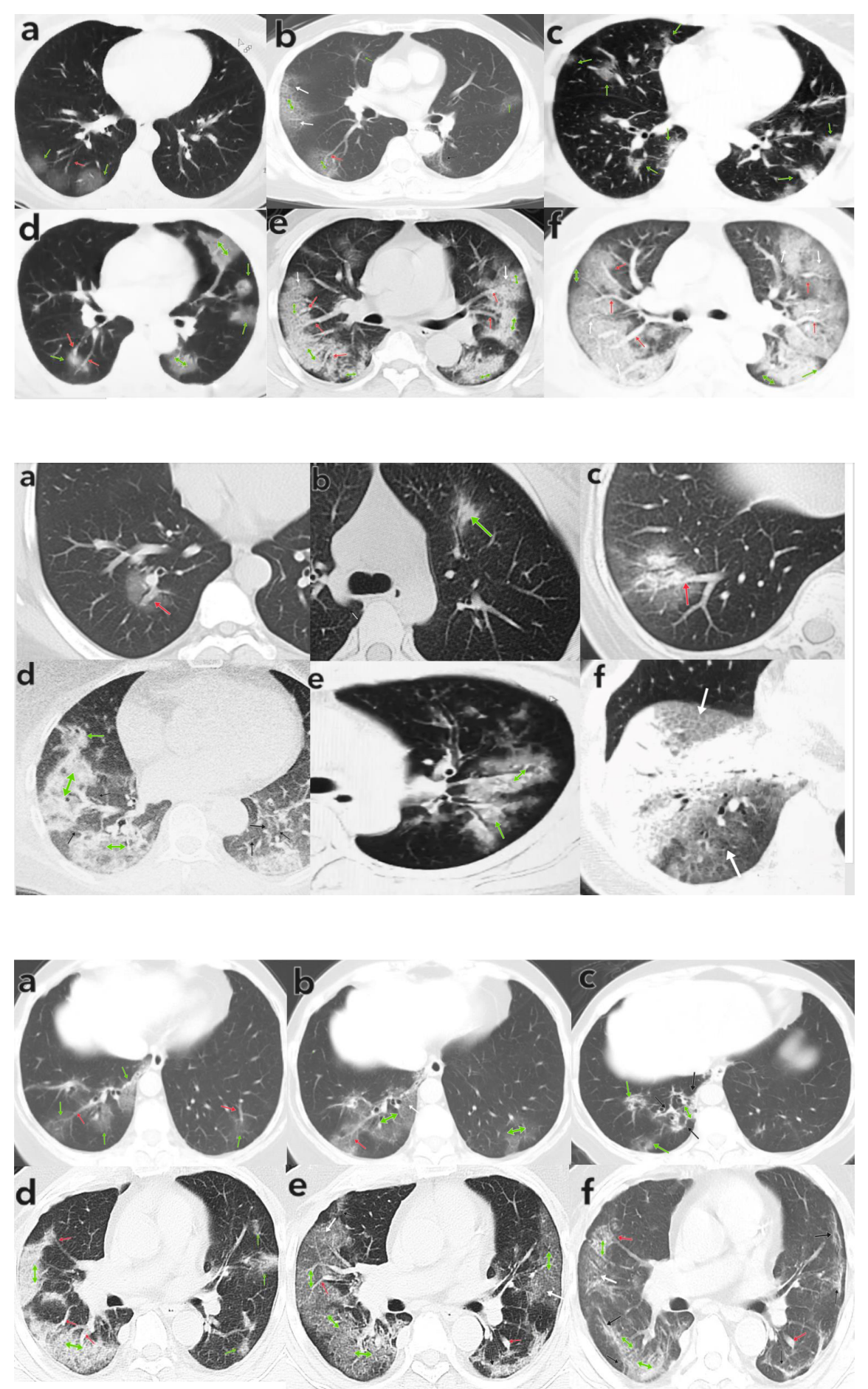


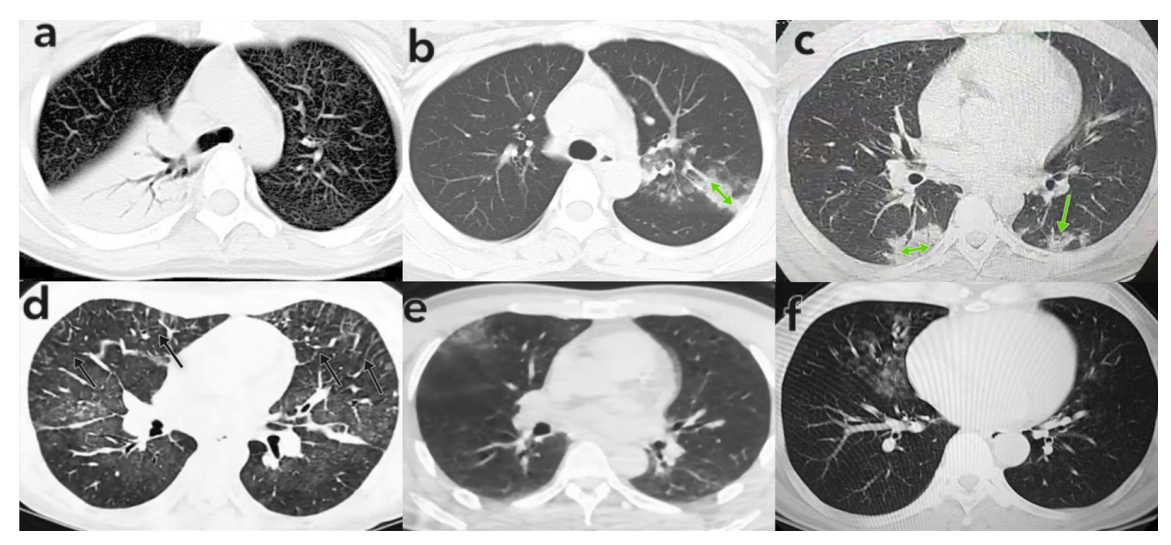

